\title{
A New Optimized Runge-Kutta Pair for the Numerical Solution of the Radial Schrödinger Equation
}

\author{
Yonglei Fang, ${ }^{1}$ Qinghong $\mathrm{Li}^{2}{ }^{2}$ Qinghe Ming, ${ }^{1}$ and Kaimin Wang ${ }^{1}$ \\ ${ }^{1}$ School of Mathematics and Statistics, Zaozhuang University, Zaozhuang 277160, China \\ ${ }^{2}$ Department of Mathematics, Chuzhou University, Chuzhou 239000, China
}

Correspondence should be addressed to Yonglei Fang, ylfangmath@gmail.com

Received 9 May 2012; Revised 23 September 2012; Accepted 9 October 2012

Academic Editor: Malisa R. Zizovic

Copyright (C) 2012 Yonglei Fang et al. This is an open access article distributed under the Creative Commons Attribution License, which permits unrestricted use, distribution, and reproduction in any medium, provided the original work is properly cited.

\begin{abstract}
A new embedded pair of explicit modified Runge-Kutta (RK) methods for the numerical integration of the radial Schrödinger equation is presented. The two RK methods in the pair have algebraic orders five and four, respectively. The two methods of the embedded pair are derived by nullifying the phase lag, the first derivative of the phase lag of the fifth-order method, and the phase lag of the fourth-order method. Nu merical experiments show the efficiency and robustness of our new methods compared with some well-known integrators in the literature.
\end{abstract}

\section{Introduction}

In molecular dynamics, quantum physics, and chemistry, no other equation has been studied more profoundly than the Schrödinger equation [1-3]. The one-dimensional Schrödinger equation has the form

$$
y^{\prime \prime}(x)=(v(x)-E) y(x)
$$

where $E$ is a real number denoting the energy, the function $v(x)$ is the effective potential satisfying $v(x) \rightarrow 0$ as $x \rightarrow \infty$. There have been a lot of numerical methods, such as exponentially fitted and phase fitted integrators based on the oscillatory property of the solution of the Schrödinger equation (1.1) (see, e.g., [4-13]). In [7], Simos and Aguiar constructed a modified Runge-Kutta method for the numerical integration of the Schrödinger equation by phase fitting based on the fifth-order RK method. Recently, Vyver improved this 
method and gave an embedded pair of modified RK methods by nullifying the dispersion (phase lag) of the fifth-order method and the fourth-order method [5].

In this paper, we derive a new kind of phase fitting RK embedded pair by nullifying the phase lag, the first derivative of phase lag of the fifth-order method, and the phase lag of the fourth-order method.

\section{Order Conditions and Phase Properties of Modified Runge-Kutta Methods}

\subsection{Modified Runge-Kutta Methods}

For the numerical integration of the initial-value problem of first-order differential equations

$$
y^{\prime}(x)=f(x, y), \quad y\left(x_{0}\right)=y_{0},
$$

we consider the s-stage modified explicit Runge-Kutta method of the form

$$
\begin{gathered}
Y_{i}=\gamma_{i} y_{n}+h \sum_{j=1}^{i-1} a_{i j} f\left(x_{n}+c_{j} h, Y_{j}\right), \quad i=1, \ldots, s, \\
y_{n+1}=y_{n}+h \sum_{i=1}^{s} b_{i} f\left(x_{n}+c_{i} h, Y_{i}\right),
\end{gathered}
$$

which can be expressed in Butcher tableau as

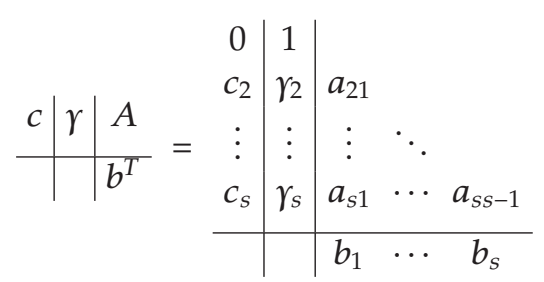

or equivalently by the triplet $(c, \gamma, A, b)$ with $c=\left(0, c_{2}, \ldots, c_{s}\right)^{T}, \gamma=\left(\gamma_{1}, \ldots, \gamma_{s}\right), A=\left(a_{i j}\right)_{s \times S}$, $b=\left(b_{1}, \ldots, c_{s}\right)$. Here, following the approach of exponential fitting and/or phase fitting in $[5,7,14]$, the frequency-depending parameters $\gamma_{i}=\gamma_{i}(v), v=h \omega, i=1, \ldots, s$ are introduced to adapt the traditional RK method to the oscillatory feature of the solution to the problem [15-27], for example, in this paper, to minimize the dispersion and/or dissipation (see next section).

The algebraic order conditions presented in [28] are not fit for the modified RK method (2.2). Writing

$$
\gamma_{i}=1+r_{i}^{(2)} v^{2}+r_{i}^{(4)} v^{4}+r_{i}^{(6)} v^{6}+\cdots,
$$

where $\gamma_{i}^{(2 j)}=\left(d^{2 j} \gamma_{i} / d v^{2 j}\right)(0), j=1,2, \ldots$, the third-to-fifth order conditions are listed as follows (see Vyver [5]). 
(i) Order 3 requires

$$
\sum_{i} b_{i} r_{i}^{(2)}=0
$$

(ii) order 4 requires, in addition

$$
\sum_{i} b_{i} c_{i} r_{i}^{(2)}=0, \quad \sum_{i, j} b_{i} a_{i j} \gamma_{j}^{(2)}=0
$$

(iii) order 5 requires, in addition

$$
\begin{aligned}
\sum_{i} b_{i}\left(r_{i}^{(2)}\right)^{2}=0, \quad \sum_{i} b_{i} r_{i}^{(4)}=0, & \sum_{i} b_{i} c_{i}^{2} r_{i}^{(2)}=0, \\
\sum_{i j} b_{i} c_{i} a_{i j} r_{j}^{(2)}=0, \quad \sum_{i j} b_{i} a_{i j} c_{j} r_{j}^{(2)}=0, & \sum_{i j} b_{i} a_{i j} a_{j k} r_{k}^{(2)}=0 .
\end{aligned}
$$

\subsection{Dispersion and Dissipation of Modified Runge-Kutta Methods}

Applying the modified RK method (2.2) to the test differential equation

$$
y^{\prime}=\mathrm{i} \omega y, \quad \omega>0,
$$

yields

$$
y_{n+1}=R(\mathrm{i} v) y_{n}, \quad v=h w,
$$

where

$$
\begin{aligned}
R(\mathrm{i} v) & =1-\mathrm{i} v b^{T}(I-\mathrm{i} v A)^{-1} \gamma \\
& =1-\mathrm{i} v b^{T} \gamma+v^{2} b^{T} A \gamma+\mathrm{i} v^{3} b^{T} A^{2} \gamma-\cdots+(\mathrm{i} v)^{S} b^{T} A^{s-1} \gamma
\end{aligned}
$$

with $I$ the $s \times s$ identity matrix.

Definition 2.1. The quantities

$$
P(v)=v-\arg (R(\mathrm{i} v)), \quad D(v)=1-|R(\mathrm{i} v)|,
$$


are called the dispersion (or phase lag) and the dissipation (or amplification factor error) of the method (2.2), respectively. And the method is said to be dispersive of order $q$ and dissipative of order $r$ if

$$
P(v)=\mathcal{O}\left(v^{q+1}\right), \quad D(v)=\mathcal{O}\left(v^{r+1}\right),
$$

respectively.

Writing

$$
R(v)=U\left(v^{2}\right)+\mathrm{i} v V\left(v^{2}\right)
$$

we have

$$
P(v)=v-\arctan \left(v \frac{V\left(v^{2}\right)}{U\left(v^{2}\right)}\right), \quad D(v)=1-\sqrt{U^{2}\left(v^{2}\right)+v^{2} V^{2}\left(v^{2}\right)}
$$

From the formula (2.10), $U\left(v^{2}\right)$ and $V\left(v^{2}\right)$ are polynomials in $v^{2}$ :

$$
\begin{gathered}
U\left(v^{2}\right)=1-v^{2} b^{T} A \gamma+v^{4} b^{T} A^{3} \gamma-\cdots, \\
V\left(v^{2}\right)=b^{T} \gamma-v^{2} b^{T} A^{2} \gamma+v^{4} b^{T} A^{4} \gamma-\cdots,
\end{gathered}
$$

which are completely determined by $c, A, \gamma$, and $b$.

\section{Construction of the New Embedded Pair}

In this section, we are concerned with the embedded modified pair which is simplify denoted by the Butcher tableau

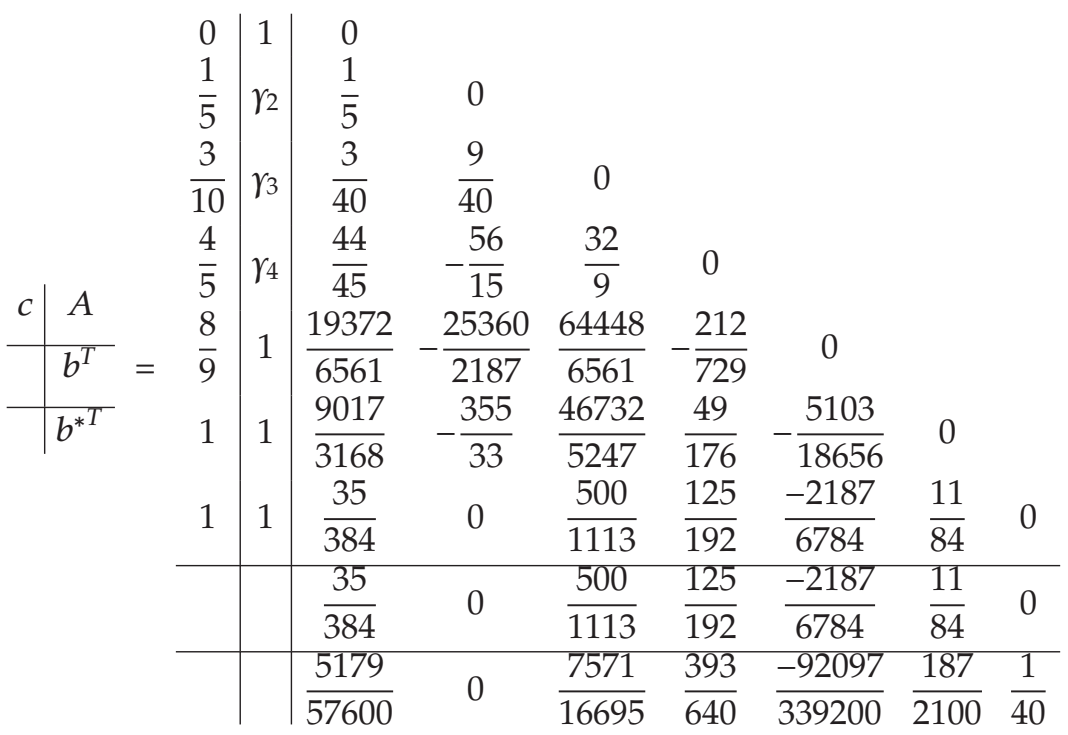


Choosing $\gamma_{2}=\gamma_{3}=\gamma_{4}=1$, the classical embedded RK5(4) pair derived by Dormand and Prince [29] is recovered, where the method $(c, A, b)$ is of order five and $\left(c, A, b^{*}\right)$ is of order four. It should be noted that the first method $(c, A, b)$ in this pair shares the FSAL property in the sense that it uses only six function evaluations at each step with seven stages. Simos and Aguiar [7] presented a modified phase fitted RK method by determining the one-parameter $\gamma_{2}$. Following this approach, Vyver [5] constructed a phase fitted embedded RK5(4) pair. Our main aim in this section is to derive a more efficient embedded RK pair.

Inspired by the ideas in [30-40], with a variant expression of dispersion (see Simos [41]), we compute the dispersion of the higher-order method and the dispersion of the lowerorder method and the first derivative of dispersion of the higher-order method of the pair (3.1) as follows:

$$
\begin{gathered}
P_{H}(v)=\tan (v)-\frac{M_{1}}{N_{1}}, \\
P_{L}(v)=\tan (v)-\frac{M_{2}}{N_{2}}, \\
\operatorname{Der} P_{H}(v)=\sec ^{2}(v)-\frac{M_{1}^{\prime} N_{1}-N_{1}^{\prime} M_{1}}{N_{1}^{2}},
\end{gathered}
$$

in which

$$
\begin{aligned}
M_{1}= & 15 v\left(107145+48230 v^{2}-8904 \gamma_{2} v^{4}+5565 \gamma_{4}\left(2 v^{2}-125\right)+320 \gamma_{3}\left(371 v^{2}-1500\right)\right), \\
N_{1}= & 7\left(225\left(563+3200 \gamma_{3}+1325 \gamma_{4}\right) v^{2}+10600\left(3 \gamma_{2}-4-8 \gamma_{3}\right) v^{4}+3816 v^{6}-2289600\right), \\
M_{2}= & v\left(216532500+173461225 v^{2}-113080800 \gamma_{2} v^{2}-9283904 v^{4}-17051160 \gamma_{2} v^{4}\right. \\
& \left.+133560 v^{6}+83475 \gamma_{4}\left(397 v^{2}-23580\right)-8000 \gamma_{3}\left(181704-55090 v^{2}+371 v^{4}\right)\right), \\
N_{2}= & -3205440000+75\left(2536615+13703168 \gamma_{3}+5129817 \gamma_{4}\right) v^{2} \\
& -3710\left(9263+2352 \gamma_{2}+24160 \gamma_{3}+225 \gamma_{4}\right) v^{4}+26712\left(136+25 \gamma_{2}\right) v^{6} .
\end{aligned}
$$

Solving the systems of (3.2) we obtain

$$
\begin{aligned}
\gamma_{2}=(-25 v( & \left.102879000-16468980 v^{2}+5693624 v^{6}+52050 v^{8}\right) \cos (v)+4473000000 \cos (v)^{2} \sin (v) \\
+ & v\left(-25\left(76041000-26581020 v^{2} 3569296 v^{4}+469645 v^{6}\right) \cos (3 v)\right. \\
- & 2 v\left(1225233750+380478750 v^{2}+59883450 v^{4}-19537525 v^{6}-233289 v^{8}+3710 v^{10}\right) \\
& \left.\left.\times \sin (v)-50 v\left(46352550-1042218 v^{2}-804802 v^{4}+97573 v^{6}\right) \sin (3 v)\right)\right) /\left(5 v^{4} M\right),
\end{aligned}
$$




$$
\begin{aligned}
& r_{3}=(v(211041495000 \\
& \left.+v^{2}\left(-11689730450+v^{2}\left(-1264487200+7 v^{2}\left(58586875+212 v^{2}\left(45 v^{2}-8753\right)\right)\right)\right)\right) \\
& \times \cos (v)-212026500000 \cos (v)^{2} \sin (v) \\
& +5 v\left(-5\left(-39400200-181538138 v^{2}+7037128 v^{4}+878157 v^{6}\right) \cos (v)\right. \\
& -2 v\left(7258856775+v^{2}\left(-1213296080+7 v^{2}\left(17587275+53 v^{2}\left(480 v^{2}-56077\right)\right)\right)\right) \\
& \left.\left.\times \sin (v)-10 v\left(149294235-22496216 v^{2}+2498685 v^{4}\right) \sin (3 v)\right)\right) /\left(200 v^{2} M\right) ， \\
& \gamma_{4}=\left(-v\left(14108160000-5363145725 v^{2}+258455175 v^{4}+22475450 v^{6}-6105436 v^{8}+89040 v^{10}\right)\right. \\
& \times \cos (v)+18288000000 \cos (v)^{2} \sin (v) \\
& +v\left(-25\left(167193600+12945389 v^{2}-2421645 v^{4}+68704 v^{6}\right) \cos (3 v)\right. \\
& -2 v\left(2084367750+552861425 v^{2}-100100925 v^{4}+15041455 v^{6}-1192856 v^{8}+14840 v^{10}\right) \\
& \left.\left.\times \sin (v)+50 v\left(-6590070-3738885 v^{2}+176272 v^{4}\right) \sin (3 v)\right)\right) /\left(25 v^{2} M\right),
\end{aligned}
$$

where

$$
\begin{aligned}
M=\left(v\left(2013125+23057425 v^{2}-1145578 v^{4}+7420 v^{6}\right) \cos (v)+200850000 \cos (v)^{2} \sin (v)\right. \\
-25 v\left(\left(1059125-97573 v^{2}\right) \cos (3 v)+2 v\left(2650105-310653 v^{2}+1855 v^{4}\right) \sin (v)\right. \\
+389770 v \sin (3 v))) .
\end{aligned}
$$

For small values of $v$, say $|v|<0.04$, the above formulae are subject to heavy cancelations and in that case the following Taylor series expansions must be used

$$
\begin{aligned}
\gamma_{2}= & 1-\frac{22051 v^{2}}{1175900}-\frac{23696602511 v^{4}}{10453520523600}-\frac{1936263085085455799 v^{6}}{921922108777593000000} \\
& -\frac{132294694246155651595747 v^{8}}{112745173602003447304800000}+\cdots,
\end{aligned}
$$




$$
\begin{aligned}
r_{3}= & 1-\frac{28991 v^{4}}{5879500}+\frac{2740771225019 v^{6}}{1866700093500000}-\frac{633801949482499571 v^{8}}{1317031583967990000000} \\
& -\frac{708641114757728596912360427 v^{10}}{211397200503756463696500000000}+\cdots, \\
r_{4}= & 1+\frac{35008 v^{4}}{10289125}-\frac{77729377736 v^{6}}{408340645453125}-\frac{1838102360373886 v^{8}}{8002796083138828125} \\
& -\frac{5030427774325408868897437 v^{10}}{19267974004248636014003906250}+\cdots
\end{aligned}
$$

It is easy to check that the two schemes in the new pair (3.1) with $\gamma$-values (3.5) are of algebraic orders five and four, respectively. The Taylor series of the dissipations of the new fifth-order method and the fourth-order method are given by

$$
\begin{gathered}
D_{H}(v)=-\frac{215377 v^{6}}{197551200}+\frac{637415987609 v^{8}}{9408168471240000}-\frac{4487714698722250553 v^{10}}{66378391831986696000000}+\cdots \\
D_{L}(v)=-\frac{10822793 v^{6}}{9260212500}+\frac{7211815545941 v^{8}}{261338013090000000}-\frac{154473286018569080117 v^{10}}{1382883163166389500000000}+\cdots
\end{gathered}
$$

respectively.

\section{Stability Analysis}

In this section, we are interested in the phase properties of the new methods. Lambert and Watson's stability theory [42] was reconsidered by Coleman and Ixaru [43] for the periodicity of exponentially-fitted symmetric methods for $y^{\prime \prime}=f(x, y)$. Vyver [44] formulated this theory to RK methods. Following Van de Vyver's idea, we consider the test equation

$$
y^{\prime}=i \lambda y, \quad \lambda>0
$$

Applying the modified RK method (3.1) to test (4.1) yields the difference equation

$$
y_{n+1}=M(\mathrm{i} \theta, v) y_{n}, \quad \theta=\lambda h,
$$

where

$$
M(\mathrm{i} \theta, v)=\frac{\operatorname{det}\left(I-\mathrm{i} \theta A+\mathrm{i} \theta \gamma(v) b^{T}\right)}{\operatorname{det}(I-\mathrm{i} \theta A)}
$$

with $I$ the $s \times s$ identity matrix. 
Definition 4.1. For the modified RK method (3.1) with stability function $M(i \theta, v)$, the quantities

$$
\widetilde{P}(\theta, v)=\theta-\arg (M(\mathrm{i} \theta, v)), \quad \widetilde{D}(\theta, v)=1-|M(\mathrm{i} \theta, v)|
$$

are called the phase lag (dispersion) and amplification factor error (dissipation), respectively. If

$$
\widetilde{P}(\theta, v)=c_{\phi} \theta^{q+1}+\mathcal{O}\left(\theta^{q+3}\right), \quad \tilde{D}(\theta, v)=c_{d} \theta^{p+1}+\mathcal{O}\left(\theta^{p+3}\right),
$$

the method is said to be of phase lag order $q$ and dissipation order $p$, respectively, where the $c_{\phi}$ and $c_{d}$ are called the phase lag constant and dissipation constant, respectively.

For the convenience of analyzing the phase lag and the dissipation, we denote the ratio $r=v / \theta$. Then the phase lags and the dissipations of the higher-order method and the lower order method are

$$
\begin{gathered}
\tilde{P}_{H}(\theta, r \theta)=-\frac{\left(r^{2}-1\right)^{2}\left(176385+198413 r^{2}\right)}{370408500} \theta^{7}+\mathcal{O}\left(\theta^{9}\right), \\
\tilde{D}_{H}(\theta, r \theta)=\frac{\left(164626-154357 r^{2}-656400 r^{4}\right)}{592653600} \theta^{6}+\mathcal{O}\left(\theta^{8}\right), \\
\tilde{P}_{L}(\theta, r \theta)=-\frac{\left(r^{2}-1\right)\left(3421869+621392 r^{2}\right)}{4233240000} \theta^{5}+\mathcal{O}\left(\theta^{7}\right), \\
\tilde{D}_{L}(\theta, r \theta)=\frac{\left(-31690505+26395047 r^{2}-37995714 r^{4}\right)}{37040850000} \theta^{6}+\mathcal{O}\left(\theta^{8}\right),
\end{gathered}
$$

respectively. Thus, the higher-order method has a phase lag of order six and a dissipation of order five and the low-order method is of phase lag order four and dissipation order five.

\section{Numerical Experiments}

In this section, we will compare the numerical performance of the new pair with some existing well-known methods proposed in the scientific literature.

\subsection{Comparison with Fixed Step-Size Methods}

The following fixed step-size methods are selected for comparison:

(i) PHARK5S: the phase fitted fifth-order RK method derived by Simos [6];

(ii) MODPHARK5S: the modified phase fitted fifth-order RK method obtained by Simos and Aguiar in [7];

(iii) MODPHARK5V: the higher-order method of the modified phase fitted embedded RK5(4) pair obtained by Vyver presented in [5]; 


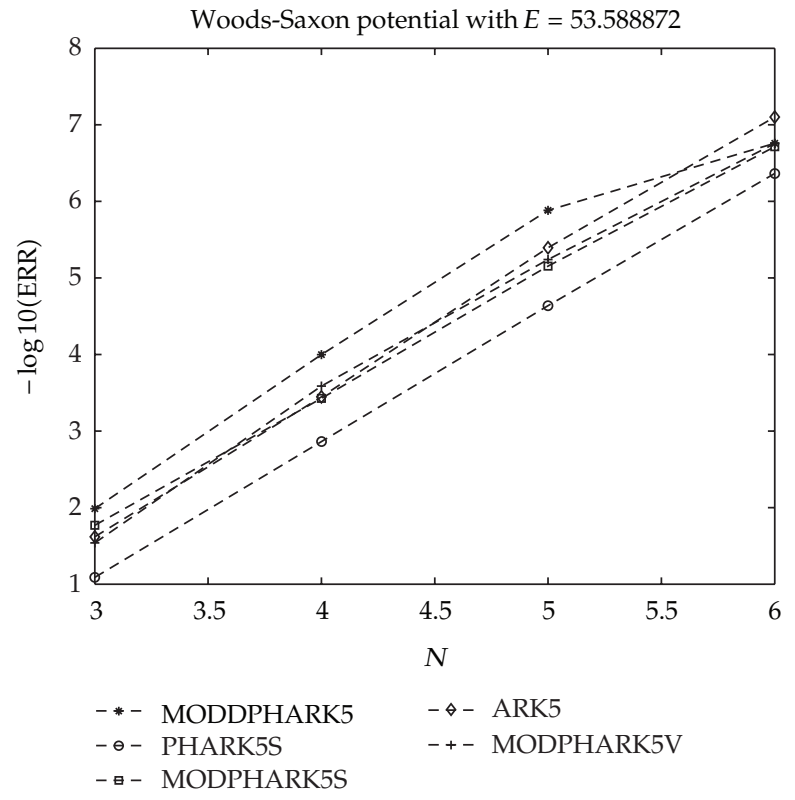

Figure 1: Efficiency curves for $E=53.588872$.

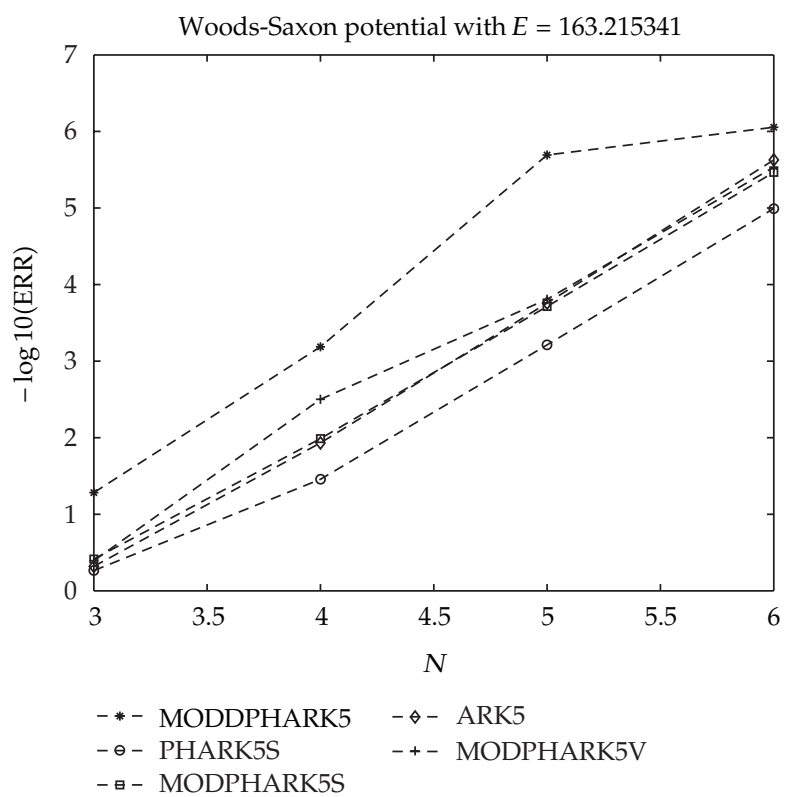

Figure 2: Efficiency curves for $E=163.215341$.

(iv) ARK5: an adapted fifth-order RK method given by Fang et al. in [45];

(v) MODDPHARK5: the higher-order method of the new pair. 


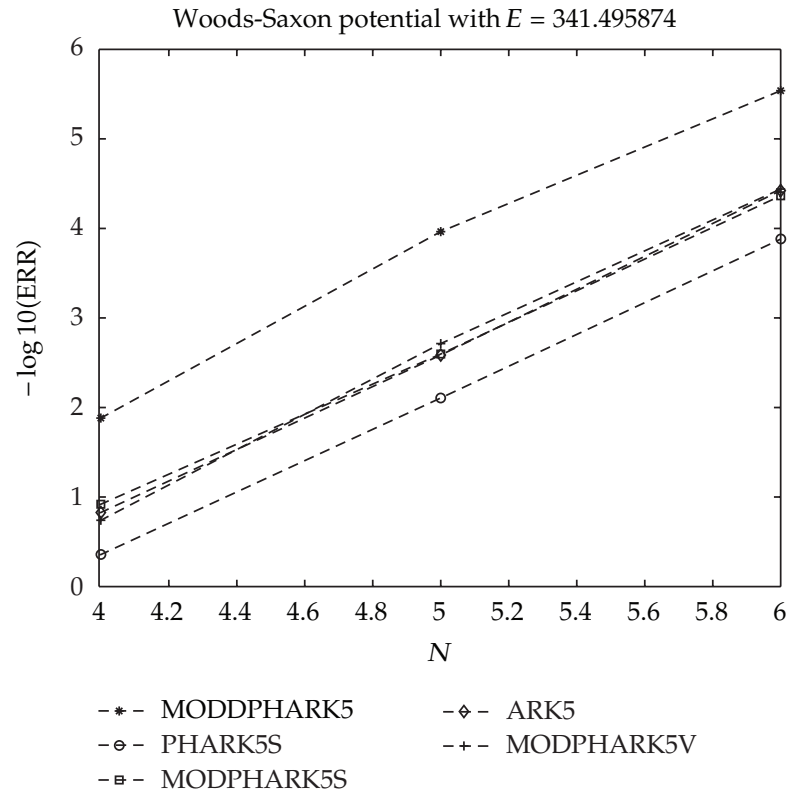

Figure 3: Efficiency curves for $E=341.495874$.

We consider the numerical integration of the Schrödinger equation (1.1) with the well-known Woods-Saxon potential

$$
v(x)=c_{0} z(1-a(1-z)),
$$

in which $z=(\exp (a(x-b)+1))^{-1}, c_{0}=-50, a=5 / 3$, and $b=7$. The domain of numerical integration is $[0,15]$. It is appropriate to choose $\omega$ as follows $[5,46]$ :

$$
\omega= \begin{cases}\sqrt{50+E}, & x \in[0,6.5] \\ \sqrt{E}, & x \in[6.5,15]\end{cases}
$$

In the numerical experiments we consider the resonance problem $(E>0)$, the numerical results were compared with the analytical solution of the Woods-Saxon potential, rounded to six decimal places. In Figures 1, 2, 3, and 4, we plot the error of $-\log _{10}\left|E_{\text {analytical }}-E_{\text {calculated }}\right|$ versus the integration step-size $\left(1 / 2^{N}\right)$ for $E_{\text {analytical }}=$ 53.588872,163.215341,341.495874, and 989.701916, respectively.

\subsection{Comparison with Variable Step-Size Methods}

Next we select the following embedded RK5(4) pairs:

(i) PHARK5(4)S: the phase fitted embedded RK5(4) pair derived by Simos [6];

(ii) MODPHARK5(4)V: the modified phase fitted embedded RK5(4) pair obtained by Vyver presented in [5];

(iii) MODDPHARK5(4): the new embedded RK5(4) pair given in this paper. 


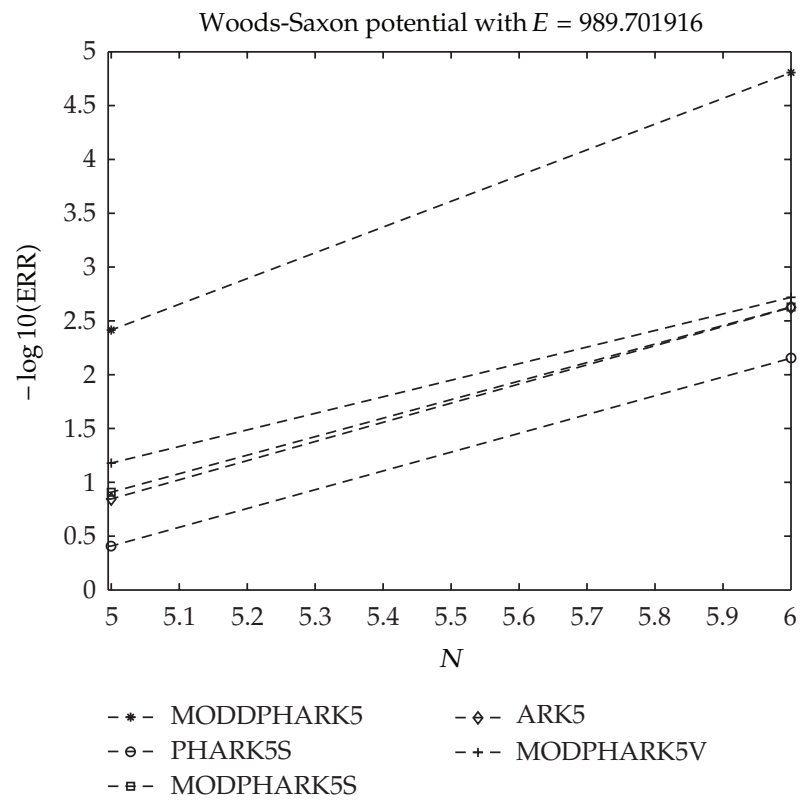

Figure 4: Efficiency curves for $E=989.701916$.

We consider the numerical integration of the Schrödinger equation (1.1) with the well-known Lennard-Jones potential (see [5])

$$
v(x)=\frac{l(l+1)}{x^{2}}+500\left(\frac{1}{x^{12}}-\frac{1}{x^{6}}\right)
$$

and we compute some phase shifts for this potential. In the numerical results, we compute the phase shifts correct to four decimal places for the energies $k^{2}=2500$ and $k^{2}=10000$. We choose the fitting frequency $\omega=k$. For the calculation of the phase shifts, we show the number of function evaluations as a function of $l=0,1, \ldots, 10$ in Figures 5-6.

Figures 1-6 show that our new methods are more efficient than the other methods we select for comparison.

\section{Conclusions and Discussions}

A new kind of modified phase fitted explicit embedded RK pair for the numerical integration of the radial Schrödinger equation is presented in this paper. This new pair is based on the classical RK5(4) pair obtained by Dormand and Prince [29]. The phase fitted technique can be regarded as an improvement of the ideas from $[5,7,30,31]$. The two schemes in this pair are of orders five and four, respectively. Numerical experiments show the effectiveness and competence of the new pair. 


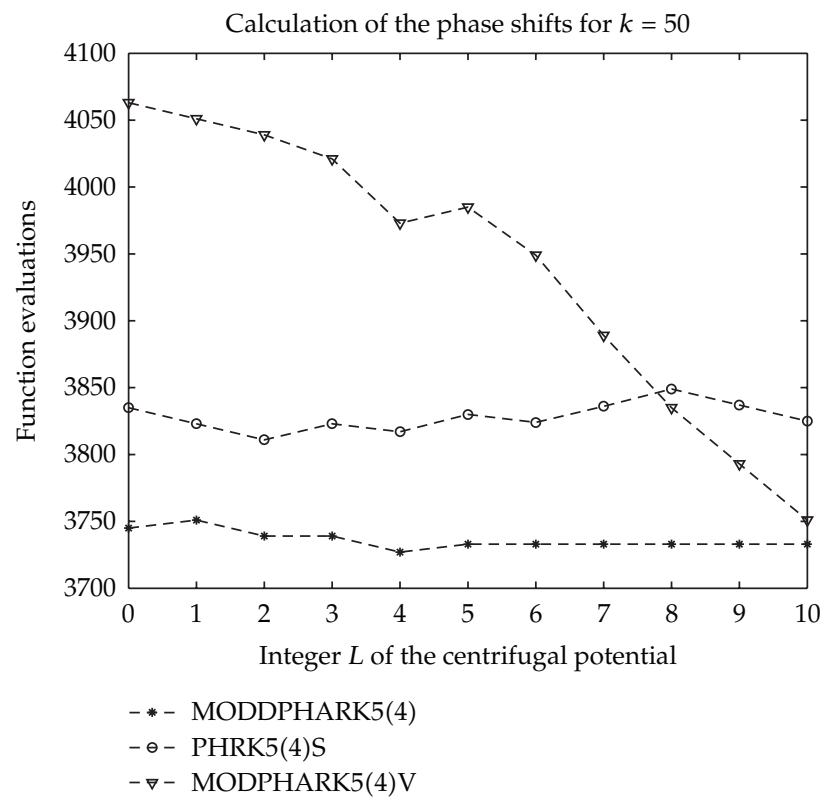

Figure 5: Efficiency curves for $k=50$.

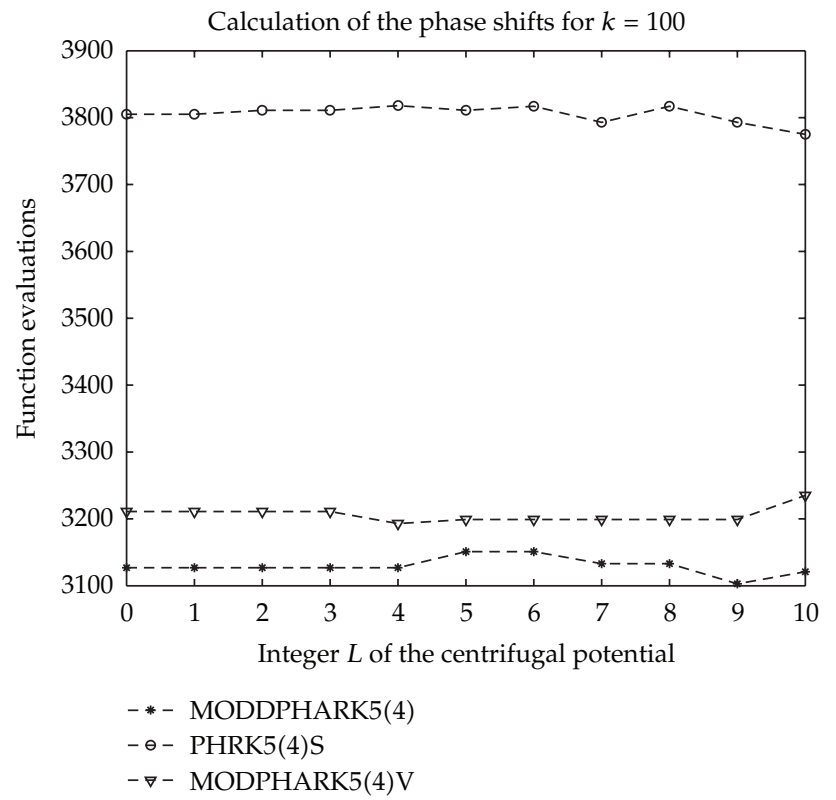

Figure 6: Efficiency curves for $k=100$.

\section{Acknowledgments}

The authors are deeply grateful to the anonymous referees for their constructive comments and valuable suggestions. This research is partially supported by NSFC (no. 11101357), the foundation of Shandong Outstanding Young Scientists Award Project (no. BS2010SF031), the 
foundation of Scientific Research Project of Shangdong Universities (no. J11LG69), NSF of Shandong Province, China (no. ZR2011AL006), NSF of Universities of Anhui Province, China (no. KJ2010A248), and the Scientific Research Start-up Fund of Chuzhou University, China (no. 2010qd03).

\section{References}

[1] A. C. Allison, "The numerical solution of coupled differential equations arising from the Schrödinger equation," Journal of Computational Physics, vol. 6, pp. 378-391, 1970.

[2] J. M. Blatt, "Practical points concerning the solution of the Schrödinger equation," Journal of Computational Physics, vol. 1, pp. 378-391, 1961.

[3] J. W. Cooley, "An improved eigenvalue corrector formula for solving the Schrödinger equation for central fields," Mathematics of Computation, vol. 15, pp. 363-374, 1961.

[4] T. E. Simos, "A family of fifth algebraic order trigonometrically fitted Runge-Kutta methods for the numerical solution of the Schrödinger equation," Computational Materials Science, vol. 34, no. 4, pp. 342-354, 2005.

[5] H. Van De Vyver, "An embedded phase-fitted modified Runge-Kutta method for the numerical integration of the radial Schrödinger equation," Physics Letters A, vol. 352, no. 4-5, pp. 278-285, 2006.

[6] T. E. Simos, "An embedded Runge-Kutta method with phase-lag of order infinity for the numerical solution of the Schrödinger equation," International Journal of Modern Physics C, vol. 11, no. 6, pp. 1115-1133, 2000.

[7] T. E. Simos and J. V. Aguiar, "A modified phase-fitted Runge-Kutta method for the numerical solution of the Schrödinger equation," Journal of Mathematical Chemistry, vol. 30, no. 1, pp. 121-131, 2001.

[8] T. E. Simos, "Exponentially and trigonometrically fitted methods for the solution of the Schrödinger equation," Acta Applicandae Mathematicae, vol. 110, no. 3, pp. 1331-1352, 2010.

[9] Z.A. Anastassi and T.E. Simos, "A parametric symmetric linear four-step method for the efficient integration of the Schrödinger equation and related oscillatory problems," Journal of Computational and Applied Mathematics, vol. 236, no. 16, pp. 3880-3889, 2012.

[10] D. P. Sakas and T. E. Simos, "Multiderivative methods of eighth algebraic order with minimal phaselag for the numerical solution of the radial Schrödinger equation," Journal of Computational and Applied Mathematics, vol. 175, no. 1, pp. 161-172, 2005.

[11] Z. Kalogiratou, Th. Monovasilis, and T. E. Simos, "Symplectic integrators for the numerical solution of the Schrödinger equation," Journal of Computational and Applied Mathematics, vol. 158, no. 1, pp. 83-92, 2003, Selected papers from the Conference on Computational and Mathematical Methods for Science and Engineering (Alicante, 2002).

[12] T. E. Simos and P. S. Williams, "On finite difference methods for the solution of the Schrödinger equation," Computers and Chemistry, vol. 23, no. 6, pp. 513-554, 1999.

[13] A. Konguetsof and T. E. Simos, "A generator of hybrid symmetric four-step methods for the numerical solution of the Schrödinger equation," Journal of Computational and Applied Mathematics, vol. 158, no. 1, pp. 93-106, 2003, Selected papers from the Conference on Computational and Mathematical Methods for Science and Engineering (Alicante, 2002).

[14] G. Vanden Berghe, H. De Meyer, M. Van Daele, and T. Van Hecke, “Exponentially-fitted explicit Runge-Kutta methods," Computer Physics Communications, vol. 123, no. 1-3, pp. 7-15, 1999.

[15] A. D. Raptis and T. E. Simos, "A four-step phase-fitted method for the numerical integration of second order initial value problems," BIT, vol. 31, no. 1, pp. 160-168, 1991.

[16] Z. A. Anastassi and T. E. Simos, "Numerical multistep methods for the efficient solution of quantum mechanics and related problems," Physics Reports, vol. 482-483, pp. 1-240, 2009.

[17] Z. Kalogiratou and T. E. Simos, "Newton-Cotes formulae for long-time integration," Journal of Computational and Applied Mathematics, vol. 158, no. 1, pp. 75-82, 2003, Selected papers from the Conference on Computational and Mathematical Methods for Science and Engineering (Alicante, 2002).

[18] G. Psihoyios and T. E. Simos, "Trigonometrically fitted predictor-corrector methods for IVPs with oscillating solutions," Journal of Computational and Applied Mathematics, vol. 158, no. 1, pp. 135-144, 2003, Selected papers from the Conference on Computational and Mathematical Methods for Science and Engineering (Alicante, 2002). 
[19] T. E. Simos, I. T. Famelis, and C. Tsitouras, "Zero dissipative, explicit Numerov-type methods for second order IVPs with oscillating solutions," Numerical Algorithms, vol. 34, no. 1, pp. 27-40, 2003.

[20] T. E. Simos, "Dissipative trigonometrically-fitted methods for linear second-order IVPs with oscillating solution," Applied Mathematics Letters, vol. 17, no. 5, pp. 601-607, 2004.

[21] K. Tselios and T. E. Simos, "Runge-Kutta methods with minimal dispersion and dissipation for problems arising from computational acoustics," Journal of Computational and Applied Mathematics, vol. 175, no. 1, pp. 173-181, 2005.

[22] G. Psihoyios and T. E. Simos, "A fourth algebraic order trigonometrically fitted predictor-corrector scheme for IVPs with oscillating solutions," Journal of Computational and Applied Mathematics, vol. 175, no. 1, pp. 137-147, 2005.

[23] Z. A. Anastassi and T. E. Simos, "An optimized Runge-Kutta method for the solution of orbital problems," Journal of Computational and Applied Mathematics, vol. 175, no. 1, pp. 1-9, 2005.

[24] T. E. Simos, "Closed Newton-Cotes trigonometrically-fitted formulae of high order for long-time integration of orbital problems," Applied Mathematics Letters, vol. 22, no. 10, pp. 1616-1621, 2009.

[25] S. Stavroyiannis and T. E. Simos, "Optimization as a function of the phase-lag order of nonlinear explicit two-step P-stable method for linear periodic IVPs," Applied Numerical Mathematics, vol. 59, no. 10 , pp. 2467-2474, 2009.

[26] T. E. Simos, "New stable closed Newton-Cotes trigonometrically fitted formulae for long-time integration," Abstract and Applied Analysis, vol. 2012, Article ID 182536, 15 pages, 2012.

[27] T. E. Simos, "Optimizing a hybrid two-step method for the numerical solution of the Schrödinger equation and related problems with respect to phase-lag," Journal of Applied Mathematics, vol. 2012, Article ID 420387, 17 pages, 2012.

[28] E. Hairer, S. P. Nørsett, and G. Wanner, Solving Ordinary Differential Equations. I Nonstiff Problems, vol. 8, Springer, Berlin, Germany, 2nd edition, 1993.

[29] J. R. Dormand and P. J. Prince, "A family of embedded Runge-Kutta formulae," Journal of Computational and Applied Mathematics, vol. 6, no. 1, pp. 19-26, 1980.

[30] A. A. Kosti, Z. A. Anastassi, and T. E. Simos, "Construction of an optimized explicit Runge-KuttaNyström method for the numerical solution of oscillatory initial value problems," Computers $\mathcal{E}$ Mathematics with Applications, vol. 61, no. 11, pp. 3381-3390, 2011.

[31] A. A. Kosti, Z. A. Anastassi, and T. E. Simos, "An optimized explicit Runge-Kutta-Nyström method for the numerical solution of orbital and related periodical initial value problems," Computer Physics Communications, vol. 183, no. 3, pp. 470-479, 2012.

[32] I. Alolyan and T. E. Simos, "High algebraic order methods with vanished phase-lag and its first derivative for the numerical solution of the Schrödinger equation," Journal of Mathematical Chemistry, vol. 48, no. 4, pp. 925-958, 2010.

[33] I. Alolyan and T. E. Simos, "A new hybrid two-step method with vanished phase-lag and its first and second derivatives for the numerical solution of the Schrödinger equation and related problems," Journal of Mathematical Chemistry, vol. 50, no. 7, pp. 1861-1881, 2011.

[34] I. Alolyan and T. E. Simos, "A family of ten-step methods with vanished phase-lag and its first derivative for the numerical solution of the Schrödinger equation," Journal of Mathematical Chemistry, vol. 49, no. 9, pp. 1843-1888, 2011.

[35] I. Alolyan and T. E. Simos, "A family of eight-step methods with vanished phase-lag and its derivatives for the numerical integration of the Schrödinger equation," Journal of Mathematical Chemistry, vol. 49, no. 3, pp. 711-764, 2011.

[36] I. Alolyan and T. E. Simos, "Multistep methods with vanished phase-lag and its first and second derivatives for the numerical integration of the Schrödinger equation," Journal of Mathematical Chemistry, vol. 48, no. 4, pp. 1092-1143, 2010.

[37] I. Alolyan and T. E. Simos, "On eight-step methods with vanished phase-lag and its derivatives for the numerical solution of the Schrödinger equation," Communications in Mathematical and in Computer Chemistry, vol. 66, no. 2, pp. 473-546, 2011.

[38] T. E. Simos, "A two-step method with vanished phase-lag and its first two derivatives for the numerical solution of the Schrödinger equation," Journal of Mathematical Chemistry, vol. 49, no. 10, pp. 2486-2518, 2011.

[39] I. Alolyan and T. E. Simos, "A family of high-order multistep methods with vanished phase-lag and its derivatives for the numerical solution of the Schrödinger equation," Computers E Mathematics with Applications, vol. 62, no. 10, pp. 3756-3774, 2011. 
[40] I. Alolyan and T. E. Simos, "A new high order two-step method with vanished phase-lag and its derivatives for the numerical integration of the Schrödinger equation," Journal of Mathematical Chemistry, vol. 50, no. 9, pp. 2351-2373, 2012.

[41] T. E. Simos, "Runge-Kutta interpolants with minimal phase-lag," Computers $\mathcal{E}$ Mathematics with Applications, vol. 26, no. 8, pp. 43-49, 1993.

[42] J. D. Lambert and I. A. Watson, "Symmetric multistep methods for periodic initial value problems," Journal of the Institute of Mathematics and its Applications, vol. 18, no. 2, pp. 189-202, 1976.

[43] J. P. Coleman and L. Gr. Ixaru, " $P$-stability and exponential-fitting methods for $y^{\prime \prime}=f(x, y)$," IMA Journal of Numerical Analysis, vol. 16, no. 2, pp. 179-199, 1996.

[44] H. Van de Vyver, "Stability and phase-lag analysis of explicit Runge-Kutta methods with variable coefficients for oscillatory problems," Computer Physics Communications, vol. 173, no. 3, pp. 115-130, 2005.

[45] Y. Fang, Y. Song, and X. Wu, "New embedded pairs of explicit Runge-Kutta methods with FSAL properties adapted to the numerical integration of oscillatory problems," Physics Letters. A, vol. 372, no. 44, pp. 6551-6559, 2008.

[46] L. G. Ixaru and M. Rizea, "A numerov-like scheme for the numerical solution of the Schrödinger equation in the deep continuum spectrum of energies," Computer Physics Communications, vol. 19, no. 1, pp. 23-27, 1980. 


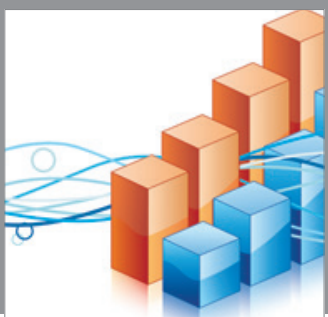

Advances in

Operations Research

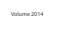

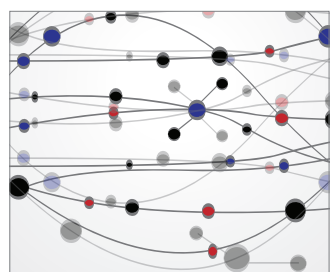

\section{The Scientific} World Journal
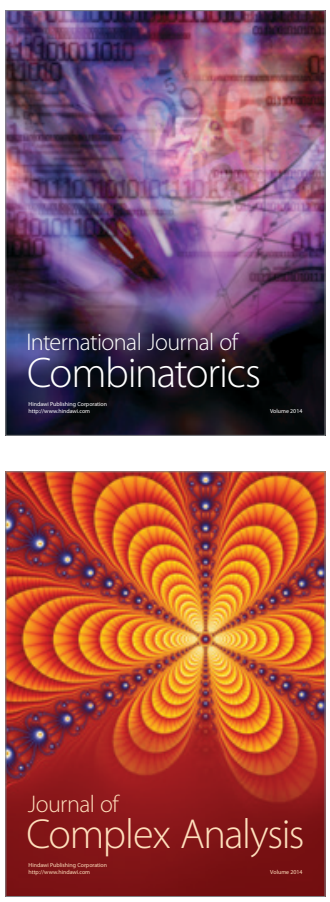

International Journal of

Mathematics and

Mathematical

Sciences
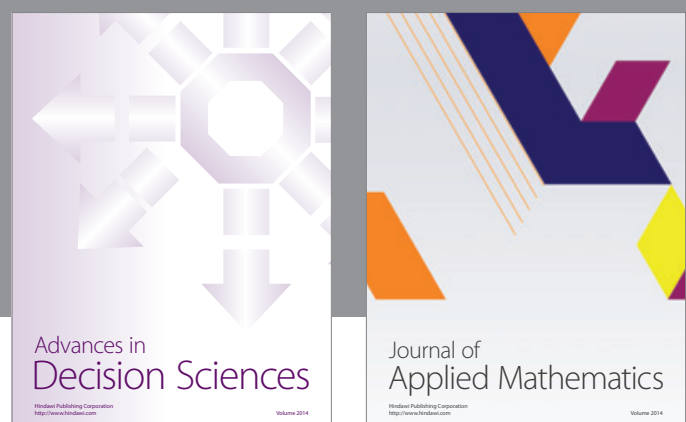

Journal of

Applied Mathematics
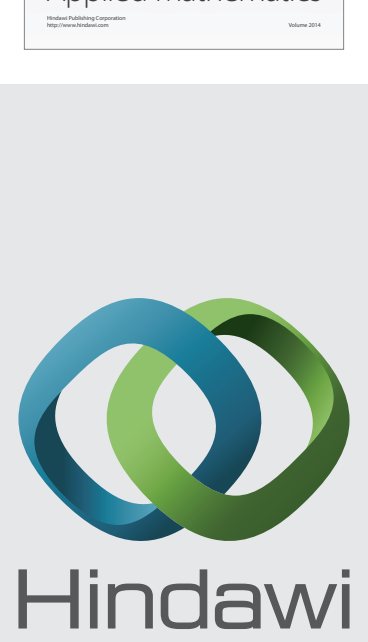

Submit your manuscripts at http://www.hindawi.com
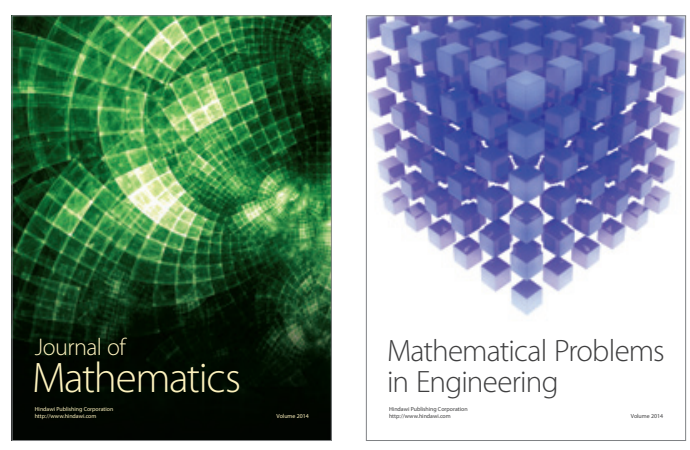

Mathematical Problems in Engineering
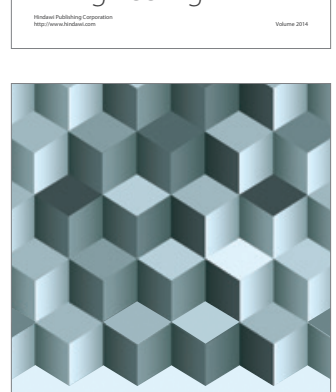

Journal of

Function Spaces
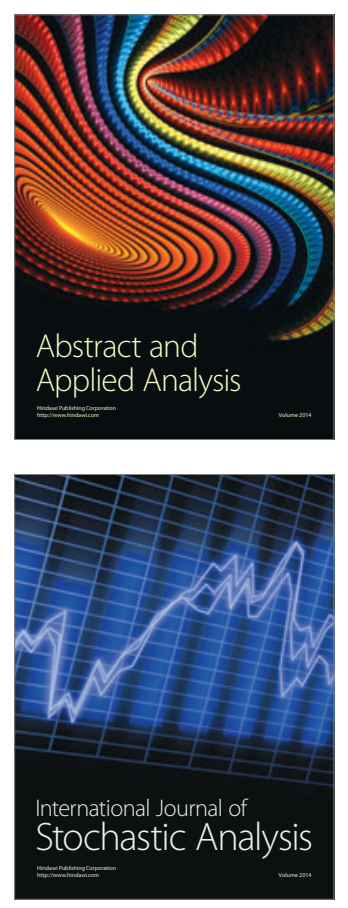

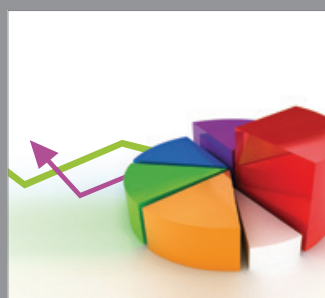

ournal of

Probability and Statistics

Promensencen
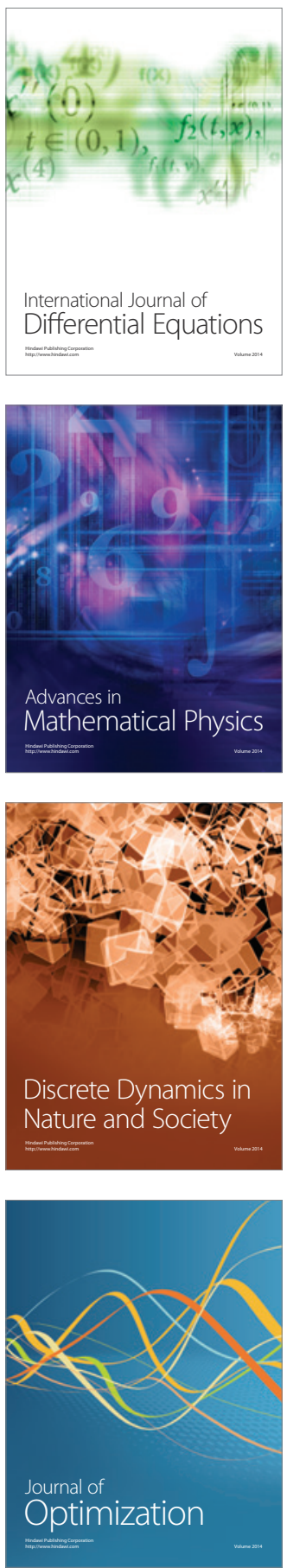